\title{
A HEURISTIC PRINCIPLE FOR A NONESSENTIAL ISOLATED SINGULARITY
}

\author{
DAVID MINDA
}

\begin{abstract}
A heuristic principle in function theory claims that a family of holomorphic (meromorphic) functions which share a property $P$ in a region $\Omega$ is likely to be normal in $\Omega$ if $P$ cannot be possessed by nonconstant entire (meromorphic) functions in the finite plane. L. Zalcman established a rigorous version of this principle. An analogous principle for a nonessential singularity is plausible: If a holomorphic (meromorphic) function $f$ has an isolated singularity at $z_{0}$, and in a deleted neighborhood of $z_{0}$ the function $f$ has a property $P$ which cannot be possessed by nonconstant entire (meromorphic) functions in the finite plane, then $z_{0}$ is a nonessential singularity. We establish a rigorous version of the principle for holomorphic functions that is very similar to Zalcman's precise statement of the other principle. -However, this rendition of the heuristic principle for a nonessential singularity fails for meromorphic functions in contrast to Zalcman's solution.
\end{abstract}

1. Introduction. L. Zalcman [11] made precise a heuristic principle in complex function theory which asserts that a family of holomorphic (meromorphic) functions which have a property $P$ in common in a region $\Omega$ is (apt to be) a normal family in $\Omega$ if $P$ cannot be possessed by nonconstant entire (meromorphic) functions in the finite plane [5, p. 250]. Let us recall Zalcman's solution. We write $\langle f, \Omega\rangle$ to denote that the function $f$ is defined on the region $\Omega \subset \mathbf{C}$. Also, $P$ will designate a property-a set - of holomorphic (meromorphic) functions. The following four conditions on $P$ play a role in Zalcman's solution.

(i) If $\langle f, \Omega\rangle \in P$ and $\Omega^{\prime} \subset \Omega$, then $\left\langle f, \Omega^{\prime}\right\rangle \in P$.

(ii) If $\langle f, \Omega\rangle \in P$ and $\phi(z)=\alpha z+\beta, \alpha \neq 0$, then $\left\langle f \circ \phi, \phi^{-1}(\Omega)\right\rangle \in P$.

(iii) Let $\left\langle f_{n}, \Omega_{n}\right\rangle \in P$, where $\Omega_{1} \subset \Omega_{2} \subset \cdots$ and $\mathbf{C}=\bigcup \Omega_{n}$. If $f_{n} \rightarrow f$ spherically uniformly on compact subsets of $\mathbf{C}$, then $\langle f, \mathbf{C}\rangle \in P$.

(iv) If $\langle f, \mathbf{C}\rangle \in P$, then $f$ is constant.

Actually, Zalcman required a slightly stronger condition than (iii), but he noted that condition (iii) as stated above is sufficient. He established the following rigorous version of the heuristic principle.

THEOREM 1. If $P$ is a property of holomorphic (meromorphic) functions which satisfies (i)-(iv), then for any region $\Omega \subset \mathbf{C}$ the family $\{f:\langle f, \Omega\rangle \in P\}$ is normal.

Received by the editors April 30, 1984.

1980 Mathematics Subject Classification. Primary 30C80.

Key words and phrases. Heuristic principle, nonessential isolated singularity, spherical derivative.

c1985 American Mathematical Society $0002-9939 / 85 \$ 1.00+\$ .25$ per page 
An analogous principle for the nonessential character of an isolated singularity of a holomorphic (meromorphic) functions seems plausible. Given $z_{0} \in \mathbf{C}$ and $r>0$, set $D\left(z_{0}, r\right)=\left\{z:\left|z-z_{0}\right|<r\right\}$ and $D^{\prime}\left(z_{0}, r\right)=D\left(z_{0}, r\right) \backslash\left\{z_{0}\right\}$. The alleged principle asserts that if $P$ is a property of holomorphic (meromorphic) functions which satisfies (i)-(iv) and $\left\langle f, D^{\prime}\left(z_{0}, r\right)\right\rangle \in P$, then $f$ has a pole or a removable singularity at the point $z_{0}$. The following two basic examples lend credence to this alleged principle.

Example 1. For fixed $M>0$ let $\langle f, \Omega\rangle \in P_{M}$ if and only if $\sup \{|f(z)|: z \in \Omega\} \leqslant$ $M$. Then (i)-(iii) are clearly satisfied. Also, if $\langle f, \mathbf{C}\rangle \in P_{M}$, then $f$ must be constant by Liouville's theorem. If $\left\langle f, D^{\prime}\left(z_{0}, r\right)\right\rangle \in P_{M}$, then $z_{0}$ is a removable singularity by a criterion of Riemann [1, p. 362].

EXAmple 2. For distinct $a, b, c \in \mathbf{C} \cup\{\infty\}$ let $\langle f, \Omega\rangle \in P_{a, b, c}$ if and only if either $f$ is constant or $f$ omits the values $a, b, c$ in $\Omega$. That conditions (i) and (ii) hold for $P_{a, b, c}$ is evident, while (iii) is a consequence of Hurwitz' theorem [1, pp. 220-221]. The fact that (iv) holds is the little Picard theorem. Finally, the big Picard theorem asserts that if $\left\langle f, D^{\prime}\left(z_{0}, r\right)\right\rangle \in P_{a, b, c}$, then $z_{0}$ is a pole or a removable singularity for $f$.

We shall see that the alleged principle is, in fact, valid for holomorphic functions but false for meromorphic functions. Both results depend on the behavior of the spherical derivative of a meromorphic function near an isolated singularity. Recall that if $f$ is holomorphic at $z_{0}$, then the spherical derivative of $f$ at $z_{0}$ is $f^{\sharp}\left(z_{0}\right)=$ $\left|f^{\prime}\left(z_{0}\right)\right| /\left(1+\left|f\left(z_{0}\right)\right|^{2}\right)$. If $f$ has a pole at $z_{0}$, then $f^{\sharp}\left(z_{0}\right)=\lim _{z \rightarrow z_{0}} f^{\sharp}(z)$. If $f$ is meromorphic on $\Omega$, then $f^{\#}$ is a continuous real-valued function on $\Omega$ [2, pp. 157, 158]. The following results are due to Lehto and Virtanen [6-8] and are basic for the remainder of this article.

THEOREM 2. (a) If $f$ is holomorphic on $D^{\prime}\left(z_{0}, r\right)$ and has an essential singularity at $z_{0}$, then $\lim \sup _{z \rightarrow z_{0}}\left|z-z_{0}\right| f^{\sharp}(z)=\infty$.

(b) If $f$ is mermorphic on $D^{\prime}\left(z_{0}, r\right)$ and has an essential singularity at $z_{0}$, then $\lim \sup _{z \rightarrow z_{0}}\left|z-z_{0}\right| f^{\sharp}(z) \geqslant 1 / 2$. Moreover, the constant $1 / 2$ is sharp.

Actually, there is a slight gap in the proof of (b) in [7, 8]; an easy correction of the error is given in [10]. Part (b) is also related to the question of the existence of a ray of Julia for a meromorphic function [9; 3, pp. 321-323]. Equality holds in (b) for the product

$$
f(z)=\prod_{n=1}^{\infty} \frac{z-z_{0}-a_{n}}{z-z_{0}+a_{n}},
$$

provided the numbers $a_{n}$ satisfy $\left|a_{n+1}\right|=o\left(\left|a_{n}\right|\right)[8]$.

2. The holomorphic function case. We now establish the alleged principle for holomorphic functions.

THEOREM 3. If $P$ is a property of holomorphic functions satisfying (i)-(iv) and $\left\langle f, D^{\prime}\left(z_{0}, r\right)\right\rangle \in P$, then $f$ has a pole or a removable singularity at $z_{0}$. 
Proof. Fix $z \in D^{\prime}\left(z_{0}, r\right)$ with $\left|z-z_{0}\right| \leqslant r / 2$. Then the disk $D\left(z,\left|z-z_{0}\right|\right)$ is contained in $D^{\prime}\left(z_{0}, r\right)$, so by condition (i), $\left\langle f, D\left(z,\left|z-z_{0}\right|\right)\right\rangle \in P$. Now, $\phi(w)=z$ $+\left|z-z_{0}\right| w$ maps $D(0,1)$ conformally onto $D\left(z,\left|z-z_{0}\right|\right)$, so condition (ii) yields $\langle f \circ \phi, D(0,1)\rangle \in P$. Theorem 1 implies that $\mathscr{G}=\{g:\langle g, D(0,1)\rangle \in P\}$ is a normal family in $D(0,1)$, so Marty's characterization of normal families [2, p. 158] provides a constant $M$ such that $g^{\sharp}(0) \leqslant M$ for all $g \in \mathscr{G}$. Since $(f \circ \phi)^{\sharp}(0)=\left|z-z_{0}\right| f^{\sharp}(z)$, we obtain

$$
\limsup _{z \rightarrow z_{0}}\left|z-z_{0}\right| f^{\sharp}(z) \leqslant M .
$$

Theorem 2(a) implies that the point $z_{0}$ is a pole or a removable singularity for $f$.

In Example 2 we noted that the property $P_{a, b, c}$ satisfied (i)-(iv). Hence, Theorem 3 shows that the big Picard theorem follows from the little Picard theorem.

3. The meromorphic function case. In this section we exhibit a property of meromorphic functions satisyfing (i)-(iv) and a function meromorphic in a punctured neighborhood of the origin which has this property and yet has an essential singularity at the origin.

Let $\Omega$ be a region in C. For $z_{0} \in \Omega$ let $\delta_{\Omega}\left(z_{0}\right)=\sup \left\{r: D\left(z_{0}, r\right) \subset \Omega\right\}$. Then $\delta_{\Omega}\left(z_{0}\right)$ is the radius of the largest disk in $\Omega$ with center $z_{0}$. Clearly, $\delta_{\mathbf{C}}=\infty$ while $\delta_{\Omega}\left(z_{0}\right)<\infty$ for all $z_{0} \in \Omega$ if $\Omega$ is a proper subregion of $\mathbf{C}$. For fixed $M>0$ we define a property $Q_{M}$ by $\langle f, \Omega\rangle \in Q_{M}$ if and only if $f^{\sharp}(z) \leqslant M / \delta_{\Omega}(z)$ for all $z \in \Omega$. We now show that $Q_{M}$ satisfies (i)-(iv). Since $\Omega^{\prime} \subset \Omega$ implies $\delta_{\Omega^{\prime}} \leqslant \delta_{\Omega}$, (i) evidently holds for $Q_{M}$. Next, assume $\langle f, \Omega\rangle \in Q_{M}$ and $\phi(z)=\alpha z+\beta, \alpha \neq 0$. Then $f \circ \phi$ is defined on $\phi^{-1}(\Omega)$. Since $(f \circ \phi)^{\#}(z)=|a| f^{\sharp}(\phi(z))$ and $|a| \delta_{\phi^{-1}(\Omega)}(z)=$ $\delta_{\Omega}(\phi(z))$, it follows that $\left\langle f \circ \phi, \phi^{-1}(\Omega)\right\rangle \in Q_{M}$. For (iii) note that $\delta_{\Omega_{n}}$ increases to $\infty$ and $f_{n}^{\#} \rightarrow f^{\sharp}$ uniformly on compact subsets of $\mathbf{C}$, so $f_{n}^{\#}(z) \leqslant M / \delta_{\Omega_{n}}(z)$ yields $f^{\#}(z) \leqslant M / \delta_{\mathbf{C}}(z)=0$, or $\langle f, \mathbf{C}\rangle \in Q_{M}$. Finally, if $\langle f, \mathbf{C}\rangle \in Q_{M}$, then $f^{\sharp}=0$ so $f$ is constant.

Next, we construct $\left\langle f, D^{\prime}(0,1)\right\rangle \in Q_{M}$ with an essential singularity at the origin. In (1) set $a_{n}=q^{n^{2}}$, where $q \in(0,1)$, and $z_{0}=0$. Then

$$
f(z)=\prod_{n=1}^{\infty} \frac{z-q^{n^{2}}}{z+q^{n^{2}}}
$$

satisfies

$$
\limsup _{z \rightarrow 0}|z| f^{\sharp}(z)=1 / 2,
$$

and $f$ is meromorphic on $\mathbf{C} \backslash\{0\}$ with an essential singularity at the origin. From (2) and the continuity of $f^{\sharp}$ on $\mathbf{C} \backslash\{0\}$, we may conclude there exists $M>0$ such that $f^{\sharp}(z) \leqslant M /|z|$ for $0<|z| \leqslant 1$. Since $\delta_{D^{\prime}(0,1)}(z) \leqslant|z|$, it follows that $\left\langle f, D^{\prime}(0,1)\right\rangle \in$ $Q_{M}$.

4. More on the property $Q_{M}$. We have just seen that property $Q_{M}$ satisfies (i)-(iv). On the other hand, we can show that any property satisfying (i)-(iv) is contained in some $Q_{M}$.

THEOREM 4. Suppose that $P$ is a property of holomorphic (meromorphic) functions satisfying (i)-(iv). Then there exists $M>0$ such that $P \subset Q_{M}$. 
Proof. Theorem 1 implies that $\mathscr{G}=\{g:\langle g, D(0,1)\rangle \in P\}$ is a normal family. Then Marty's characterization of normal families yields a positive constant $M$ such that $g^{\sharp}(0) \leqslant M$ for all $g \in \mathscr{G}$. Now, consider any region $\Omega \subset \mathbf{C}$ and $\langle f, \Omega\rangle \in P$. We shall show that $\langle f, \Omega\rangle \in Q_{M}$. If $\Omega=\mathbf{C}$, then (iv) implies that $f$ is constant, so, trivially, $0=f^{\sharp}=M / \delta_{\mathbf{C}}$. Hence, we may assume that $\Omega$ is a proper subregion of $\mathbf{C}$. Fix $z_{0} \in \Omega$ and set $\delta=\delta_{\Omega}\left(z_{0}\right)$. Then $g(w)=f\left(z_{0}+\delta w\right)$ is holomorphic (meromorphic) in $D(0,1)$, and (i) and (ii) imply that $g \in \mathscr{G}$. Hence, $\delta f^{\sharp}\left(z_{0}\right)=g^{\sharp}(0) \leqslant M$. This holds for all $z_{0} \in \Omega$, so $\langle f, \Omega\rangle \in Q_{M}$.

The following Landau type of theorem is an elementary consequence.

Corollary. Suppose $P$ is a property of holomorphic functions satisfying (i)-(iv). There exists $R=R\left(a_{0}, a_{1}, P\right)$ such that if $f(z)=a_{0}+a_{1} z+\cdots$ is holomorphic in $D(0, r), a_{1} \neq 0$ and $\langle f, D(0, r)\rangle \in P$, then $r \leqslant R$.

Proof. The theorem gives $M>0$ such that $P \subset Q_{M}$ so that $f^{\sharp}(z) \leqslant M / \delta_{D(0, r)}(z)$ for. $z \in D(0, r)$. Since $\delta_{D(0, r)}(z)=r-|z|$, we obtain

$$
\frac{\left|f^{\prime}(0)\right|}{1+|f(0)|^{2}} \leqslant \frac{M}{r}, \quad r \leqslant M\left(\frac{1+\left|a_{0}\right|^{2}}{\left|a_{1}\right|}\right)=R\left(a_{0}, a_{1}, P\right) .
$$

5. Final comments. First, we want to indicate that it is possible to formulate a valid version of the heuristic principle for meromorphic functions by adding another condition:

(v) If $\langle f, \Omega\rangle \in P$ and $\phi(\Delta)=\Omega$, where $\phi(z)=\alpha+\beta \exp (z), \beta \neq 0$, then $\langle f \circ \phi, \Delta\rangle \in P$.

If $P$ is a property of meromorphic functions satisfying (i)-(v) and $\left\langle f, D^{\prime}\left(z_{0}, r\right)\right\rangle$ $\in P$, then $f$ has a pole or a removable singularity at $z_{0}$. This can be established by considering $g(w)=f\left(z_{0}+r \exp (w)\right)$, which is meromorphic on $H=\{w: \operatorname{Re}(w)<$ $0\}$, and using the facts that $P \subset Q_{M}$ and $\delta_{H}(w)=|\operatorname{Re} w|$ to show that $\left|z-z_{0}\right| f^{\sharp}(z)$ $\rightarrow 0$ as $z \rightarrow z_{0}$. Details are left to the reader.

Second, we indicate an open problem dealing with the heuristic principle for normal families. Given a nonnegative integer $m$ and $a, b \in \mathbf{C}$ with $b \neq 0$ and also $b \neq a$ if $m=0$, define a property $Q_{a, b, m}$ of holomorphic functions by $\langle f, \Omega\rangle \in$ $Q_{a, b, m}$ if and only if either $f$ is constant or else $a \notin f(\Omega)$ and $b \notin f^{(m)}(\Omega)$, where $f^{(m)}$ denotes the $m$ th derivative of $f$. For $m=0$ we have $Q_{a, b, 0}=P_{a, b, \infty}$. That $\langle f, \mathbf{C}\rangle \in Q_{a, h, m}$ implies $f$ is constant can be found in [4, p. 60]. Thus, $Q_{a, h, m}$ satisfies (iv). Also, for any region $\Omega \subset \mathbf{C}$ the family $\left\{f:\langle f, \Omega\rangle \in Q_{a, b, m}\right\}$ is normal in $\Omega[\mathbf{1}$, p. 436]. For $m=0$ this is Montel's theorem. For $m>0$ this is a consequence of Miranda's theorem [1, pp. 417-432]. Therefore, this is an instance of the heuristic principle for normal families. However, $Q_{a, b, m}$ does not satisfy all conditions (i)-(iv). Evidently, $Q_{a, b, m}$ does satisfy (i). However, (ii) does not hold for $m>0$, since if $\phi(z)=\alpha z+\beta,(f \circ \phi)^{(m)}(z)=\alpha^{m} f^{(m)}(\phi(z))$. Thus, $(f \circ \phi)^{(m)}$ will not necessarily omit $b$ if $\alpha^{m} \neq 1$. Condition (iii) does hold for $Q_{a, b, m}$. If $\left\langle f_{n}, \Omega_{n}\right\rangle \in Q_{a, b, m}$, then Hurwitz' theorem implies that either $a \notin f(\mathbf{C})$ or else $f=a$, and that either $b \notin$ $f^{(m)}(\mathbf{C})$ or else $f^{(m)}=b$. If $f^{(m)}=b$, then $f$ is a polynomial. Since either $a \notin f(\mathbf{C})$ or 
else $f=a$, this polynomial is constant. Thus, $f^{(m)}=b \neq 0$ is impossible if $m>0$, and for $m=0$ we have $f=b$. In any case, $\langle f, \mathbf{C}\rangle \in Q_{a, b, m}$. We have just seen that $Q_{a, b, m}$ satisfies (i), (iii), and (iv) but not (ii). Thus, $Q_{a, b, m}$ is an example of a property that works in the heuristic principle for normal families, but not the rigorous version given by Zalcman in Theorem 1. Zalcman's rigorous formulation of the heuristic principle does cover the standard applications of the principle. Is it possible to find a rigorous formulation of the heuristic principle that would also work for the property $Q_{a, b, m}$ ?

\section{REFERENCES}

1. R. B. Burckel, An introduction to classical complex analysis, Vol. 1, Academic Press, New York, 1979.

2. J. Conway, Functions of one complex variable, 2nd ed., Springer-Verlag, New York, 1978.

3. A. Dinghas, Vorlesungen über Funtionentheorie, Grundlehren Math. Wiss., Bd. 110, Springer-Verlag, Berlin, 1961.

4. W. K. Hayman, Meromorphic functions, Oxford Univ. Press, Oxford, 1964.

5. E..Hille, Analyic function theory, Vol. 2, Ginn, Boston, Mass., 1962.

6. O. Lehto and K. I. Virtanen, On the behaviour of meromorphic functions in the neighbourhood of an isolated singularity, Ann. Acad. Sci. Fenn. Ser. AI 240 (1957), 9 pp.

7. O. Lehto, Distribution of values and singularities of analytic functions, Ann. Acad. Sci. Fenn. 249/3 (1957).

8. , The spherical derivative of meromorphic functions in the neighborhhod of an isolated singularity, Comment. Math. Helv. 33 (1959), 196-205.

9. J. W. Macki, On Julia's corollary to Picard's great theorem, Amer. Math. Monthly 75 (1968), 655-656.

10. D. Minda, Bloch constants for meromorphic functions near an isolated singularity, Proc. Amer. Math. Soc. 91 (1984), 69-72.

11. L. Zalcman, A heuristic principle in complex function theory, Amer. Math. Monthly 82 (1975), 813-817.

Department of Mathematical Sciences, University of Cincinnati, Cincinnati, Ohio 45221 